\title{
Appendix 2: Ortskorpus
}

Alle aufgeführten Städte wurden hinsichtlich kolonial motivierter Straßenbenennungen zwischen 1884 und 1945 untersucht. Die innerhalb des Untersuchungszeitraums erfolgten Eingemeindungen wurden berücksichtigt, werden in dieser Liste aber nicht einzeln aufgeführt.

Aachen, Aalen, Ahaus, Allenstein [Olsztyn], Altena, Altenburg, Amberg, Ansbach, Apolda, Arnstadt, Asch [Aš], Aschaffenburg, Aschersleben, Aue, Augsburg, Aussig [Ústí nad Labem], Baden-Baden, Bad Godesberg, Bad Kreuznach, Bamberg, Bautzen, Bayreuth, Bendzin [Będzin], Bergisch Gladbach, Berlin, Bernau bei Berlin, Bernburg (Saale), Beuthen [Bytom], Bielefeld, Bocholt, Bochum, Bodenbach (Sud) [Děčín], (Böhmisch-)Budweis [České Budějovice], Bonn, Bottrop, Brandenburg an der Havel, Braunsberg (Ostpreußen) [Braniewo], Braunschweig, Bremen, Bremerhaven-Wesermünde, Breslau [Wrocław], Brieg [Brzeg], Bromberg [Bydgoszcz], Brühl, Brünn [Brno], Brüx [Most], Bunzlau [Bolesławiec], Burg, Castrop-Rauxel, Celle/Zelle, Chemnitz, Krenau bzw. Chrzanow [Chrzanów], Coburg, Cottbus, Crimmitschau, Cuxhaven, Czeladz [Czeladź], Danzig [Gdańsk], Darmstadt, Datteln, Delmenhorst, Dessau, Detmold, Dinslaken, Dirschau [Tczew], Döbeln, Dombrowa [Dąbrowa Górnicza], Dortmund, Dreieich, Dresden, Duisburg, Düren, Düsseldorf, Eberswalde, Eger [Cheb], Eilenburg, Eisenach, Eisleben, Elbing [Elbląg], Elmshorn, Emden, Erfurt, Erlangen, Eschweiler, Essen, Esslingen am Neckar, Falkensee, Flensburg, Forst (Lausitz), Frankenthal (Pfalz), Frankfurt (Oder), Frankfurt am Main, Freiberg, Freiburg im Breisgau, Freital, Friedrichshafen, Fulda, Fürstenwalde/Spree, Fürth, Gablonz an der Neiße [Jablonec nad Nisou], Gelsenkirchen, Gera, Gevelsberg, Gießen, Gladbeck, Glatz [Kłodzko], Glauchau, Gleiwitz [Gliwice], Glogau [Głogów], Gnesen [Gniezno], Göppingen, Görlitz [Zgorzelec], Goslar, Gotenhafen [Gdynia], Gotha, Göttingen, Graudenz [Grudziądz], Graz, Greifswald, Greiz, Grünberg i. Schlesien [Zielona Góra], Guben [Gubin], Gumbinnen [Gussew], Gummersbach, Güstrow, Gütersloh, Hagen, Halberstadt, Halle a. d. Saale, Hallein, Hamburg, Hameln, Hamm, Hanau, Hannover, Heidelberg, Heidenheim an der Brenz, Heilbronn, Herford, Herne, Herten, Hilden, Hildesheim, Hindenburg O.S. [Zabrze], Hirschberg [Jelenia Góra], Hof, Homberg (Efze), Homburg (Saarland), Hürth, Idar-Oberstein, Iglau [Jihlava], Ingolstadt, Innsbruck, Inowrazlaw/Hohensalza [Inowrocław], Insterburg [Tschernjachowsk], Iserlohn, Itzehoe, Jägerndorf [Krnov], Jaworzno, Jena, Jungbunzlau [Mladá Boleslav], Kaiserslautern, Kalisch [Kalisz], Kamp-Lintfort, Karlsbad [Karlovy Vary], Kassel, Karlsruhe, Kattowitz [Katowice], Kempten, Kiel, Kladno [Kladno], Klagenfurt, Klausberg [Mikulczyce], Kleve, Koblenz, Kolberg [Kołobrzeg], Kolin [Kolín], 
Kolmar [Colmar], Köln, Komotau [Chomutov], Königsberg i. Pr. bzw. Königsberg (Pr.) [Kaliningrad], Konstanz, Köslin [Koszalin], Köthen (Anhalt), Krefel, Krems, Kremsier [Kroměřǐž], Küstrin [Kostrzyn nad Odrą], Kutno [Kutno], Landau in der Pfalz, Landsberg an der Warthe [Gorzów Wielkopolski], Landshut, Langenbielau [Bielawa], Leipzig, Lemberg [Lwow], Leslau [Włocławek], Leverkusen, Liegnitz [Legnica], Linz, Lippstadt, Lodz bzw. Litzmannstadt [Łódź], Lörrach, Lübeck, Luckenwalde, Lüdenscheid, Ludwigsburg, Ludwigshafen am Rhein, Lüneburg, Lünen, Magdeburg, Mährisch Ostrau [Ostrava], Mainz, Mannheim, Marburg, Marienburg [Malbork], Marienwerder [Kwidzyn], Marl, Meerane, Meiningen, Meißen, Memel [Klaipėda], Merseburg, Metz, Minden, Moers, München Gladbach bzw. Mönchengladbach, Mühlhausen/Thüringen, Mülhausen [Mulhouse], Mülheim an der Ruhr, München, Münster, Naumburg (Saale), Neisse [Nysa], Neubrandenburg, Neumünster, Neunkirchen, Neuruppin, Neuss, Neustadt an der Haardt bzw. Weinstraße, Neustadt O.S. [Prudnik], Neustrelitz, Neuwied, Nordhausen, Nordhorn, Nürnberg, Oberhausen, Offenbach am Main, Offenburg, Oldenburg, Olmütz [Olomouc], Oppeln [Opole], Oranienburg, Osnabrück, Ostrowo [Ostrów Weilkopolskiv], Pabianitz [Pabianice], Paderborn, Pardubitz [Pardubice], Passau, Pforzheim, Pilsen [Plzeň], Pirmasens, Pirna, Plauen, Posen [Poznań], Plozk [Płock], Potsdam, Prag [Praha], Prenzlau, Prerau [Přerov], Proßnitz [Prostějov], Quedlinburg, Radebeul, Rathenow, Ratibor [Racibórz], Ratingen, Ravensburg, Recklinghausen, Regensburg, Reichenbach, Reichenberg [Liberec], Remscheid, Rendsburg, Reutlingen, Rheine, Riesa, Rosenheim, Rostock, Rybnik [Rybnik], Saarbrücken, Saalfeld, Saarlouis/Saarlautern, Sagan [Żagań], Salzburg, Sankt Pölten, Schlesisch Ostrau [Slezská Ostrava], Schleswig, Schneidemühl [Piła], Schönebeck (Elbe), Schweidnitz [Świdnica], Schweinfurt, Schwelm, Schwenningen, Schwerin, Siegburg, Siegen, Soest, Solingen, Sonneberg, Sorau [Żary], Sosnowitz [Sosnowiec], Speyer, St. Ingbert, Stargard [Szczeciński], Staßfurt, Stendal, Sulzbach (Saar), Steyr, Stettin [Szczecin], Stolberg (Rhld.), Stolp [Słupsk], Stralsund, Straßburg [Strasbourg], Straubing, Stuttgart, Suhl, Swinemünde [Świnoujście], Tarnowitz [Tarnowskie Góry], Teplitz-Schönau [Teplice], Teschen [Český Těšín], Thorn [Toruń], Tilsit [Sowetsk], Trier, Troppau [Opava], Tübingen, Ulm, Velbert, Viersem, Villach, Völklingen, Waldenburg [Wałbrzych], WanneEickel, Warnsdorf [Varnsdorf], Warthenau [Zawiercie], Weiden, Wilhelmshaven, Weimar, Weißenfels, Wels, Werdau, Wernigerode, Wesel, Wiesbaden, Wetzlar, Wien, Wilhelmshaven, Wismar, Witten, Wittenberg, Wolfenbüttel, Worms, Wuppertal, Würzburg, Zdunska Wola bzw. Freihaus [Zduńska Wola], Zeitz, Zerbst/Anhalt, Zgierz [Zgierz], Zittau, Zlin [Zlín], Znaim [Znojmo], Zoppot [Sopot], Zweibrücken, Zwickau. 